\title{
Determination and Quantification of Collagen Types in Tissues Using HPLC-MS/MS
}

\author{
Statis Pataridis, Adam Eckhardt, Kateřina Mikulíková, Pavla Sedláková and Ivan Mikšík*
}

Institute of Physiology, Academy of Sciences of the Czech Republic, (v.v.i.), Vídeňská 1083, Prague 4, 142 20, Czech Republic

\begin{abstract}
A method for the determination and quantification of collagen types (I - V) using sample pretreatment has been developed. This work is a continuation of our previous work dealing with the determination of collagen types I and III in tissues [1].

The tissues (rat placenta and porcine cartilage) were firstly homogenized with a pestle in a grinding mortar with liquid nitrogen. Collagens were isolated from these tissues by cleavage with pepsin. The collagen types of interest were then precipitated successively by adding sodium chloride. For quantitation purposes, the sample preparation protocol has been simplified to the one-step precipitation of collagens from a solution containing $4.5 \mathrm{~mol} / \mathrm{L}$ of sodium chloride. The fractions were fragmented by cyanogen bromide and digested with trypsin. After that, HPLC-MS/MS (high performance liquid chromatography coupled to an ion trap mass spectrometer) analyses of the resulting peptide mixtures (peptide maps) were performed. Based on these analyses, specific (marker) peptides for each of the collagen types were selected. The marker peptides were then synthesized and used to identify and quantify the above-mentioned collagen types in tissues using HPLC-MS/MS, and for determining the limits of detection and quantification.
\end{abstract}

The applicability of this method for collagen analysis was demonstrated.

Keywords: Collagen, Collagen types, HPLC-MS/MS, Proteomics.

\section{INTRODUCTION}

Collagens are a family of extracellular matrix proteins that play a dominant role in maintaining the structure of various tissues and also have many other important functions (for example adhesion, tissue remodeling). Collagens are the most abundant proteins in the human body, constituting approximately $30 \%$ of its protein mass. Vertebrates have at least 27 collagen types with 42 distinct polypeptide chains, more than 20 additional proteins with collagen-like domains and approximately 20 isoenzymes of various collagenmodifying enzymes $[2,3]$. The most abundant collagens form extracellular fibrils or network-like structures, but the others fulfill a variety of biological functions. Fibril-forming collagens represent a set of at least nine different polypeptide chains which constitute the molecular species of type (I, II, III, V, XI, XXIV and XXVII) collagens.

Collagen type I is predominantely present as a heterotrimeric molecule, composed of two $\alpha 1(\mathrm{I})$ chains and one $\alpha 2(\mathrm{I})$ chain, $[\alpha 1(\mathrm{I})]_{2} \alpha 2(\mathrm{I})$, while the homotrimeric form $[\alpha 1(\mathrm{I})]_{3}$ has been shown to occur at low levels in normal adult skin [4]. Type I collagen is frequently accompanied by several other collagen types such as type III and type V collagen [5]. Type II collagen is present in cartilage and the vitreous humor in association with type XI collagen [6]. Type IV collagen is only found in basement membranes, where it is the major structural component.

\footnotetext{
*Address correspondence to this author at the Institute of Physiology, Academy of Sciences of the Czech Republic, Vídeňská 1083, Prague 4, Czech Republic; Tel: +420-296 44 2534; Fax: +420-296 44 2558;

E-mail: miksik@biomed.cas.cz
}

The determination of the molar ratios of particular collagen chains became of great importance due to its relationship with some deseases. For example, it has been shown that the more hydrophobic $\alpha 2$ (I) chain stabilizes the heterotrimeric form of type I collagen [7]. On the other hand the homotrimeric collagen type $\mathrm{I}$, which is composed of three $\alpha 1$ (I) chains, $[\alpha 1(\mathrm{I})]_{3}$, has been shown to be associated with certain forms of Ehlers-Danlos syndrome [8-10] and osteogenesis imperfecta $[11,12]$. It has also been reported that the presence of the homotrimeric type I collagen isotype significantly weakens the aorta [13, 14].

Some of the collagen type II peptides can serve as potential biomarkers of the activity of matrix metalloproteinases [15], which leads to the destruction of cartillage, and therefore plays an important role in osteoarthritis.

Another example of clinical importance is the determination of the collagen I/III ratio. There is a lot of information indicating that their relative proportions change under conditions of inflammation, during wound healing [16, 17], and under some pathological conditions [4, 18].

Abnormalities in the basement membrane collagen structure and function are connected to both inherited and acquired diseases.

Above are just a few examples that illustrate the great importance of the determination of collagen types in tissues. In fact, over 400 mutations in just 6 different collagens cause a variety of human diseases that include osteogenesis imperfecta, chondrodysplasias, some forms of osteoporosis, some forms of osteoarthritis, and the renal disease known as Alport syndrome [2]. Considering all of the above-mentioned 
examples, it is not surprising that there is a desire for a method which would allow us to determine and quantitate various collagen types or collagen chains.

While, there are many papers available dealing with the determination of water soluble proteins, the quantification of insoluble proteins such as collagens is usually accompanied by a lot of problems arising from the difficulties in sample preparation.

To date, several ways of quantifying collagen and/or collagen types or even particular collagen chains have been published such as the quantitation of hydroxyproline, which accounts for approximately $10 \%$ of the collagen molecule [19-21] or a method based on a spectrophotometric assay of collagen stained by a strong anionic dye (Sirius red F3BA) in picric acid solution [22-24]. However, the specificity of these methods is at least disputable.

Some of the more sophisticated methods include the radioactive labeling of proline and enzyme immunoassays using antibodies specific to each collagen type. These methods are very specific, but also very expensive and require many sample handling steps [25]. Other options which enable scientists to determine the molar ratios of particular collagen types employ the separation of the peptide mixture produced by enzymatic digestion by various separation methods, such as CE, SDS-PAGE or HPLC [26] and their detection by UV, mass spectrometry (MS) or tandem mass spectrometry (MS/MS) [1,27].

In this study, we employed the HPLC-MS/MS analysis of specific marker peptides in the peptide mixture produced by cyanogen bromide / trypsin digestion. This approach was originally utilized by us for determining collagen types I and III in skin, tendon and aorta [1]. However, the usability of the above-mentioned method was limited to those tissues where a higher collagen content could be expected. Tissues with a lower collagen content require pre-concentration of the sample prior to its analysis. Therefore, an important step in the method presented here is the sample preparation (precipitation by salt), which pre-concentrates the sample, so that it is possible to determine collagen types in tissues even when direct cleavage by cyanogen bromide / trypsin is not applicable.

\section{MATERIALS AND METHODS}

\subsection{Calibration Standards and Chemicals}

Three of the peptides used for calibration (GSEGPQ GVR, GDQGPVGR, GGAGPPGPEGGK, where $\underline{\mathbf{P}}$ represents hydroxylated proline) were synthesized by Vidia (Jesenice u Prahy, Czech Republic), the other five (GPAGPQ GPR, VGAPGPAGAR, TGPAGAAGAR, GPPGGVGFPG $\mathrm{SR}$, and GPEGPQGQR) were synthesized by Peptide 2.0 (Chantilly, VA, USA). The internal standard - tryptophanylglycine $(\mathrm{WG})$, cyanogen bromide $(\mathrm{CNBr})$, formic acid, and trypsin (lot 51K72501) were obtained from Sigma (St. Louis, MO, USA). The water used in the experiments was MilliQ, acetonitrile (HPLC gradient grade) was obtained from Merck (Darmstadt, Germany).

\subsection{Tissues and Animals Used}

Two collagen sources were used for both the isolation of collagens and their analyses. Rat placenta (two samples marked as A and B) obtained from Wistar strain rats bred in the Department of Physiology, 2nd Medical Faculty, Charles University (Prague, Czech Republic) was used for the identification of collagen types I, III, IV and V. Porcine cartilage served as a source of collagen II.

\subsection{Sample Preparation}

\subsubsection{Identification of Marker Peptides}

Placenta samples: The tissues were lyophilized and homogenized using a mortar and pestle. The resulting powder was then processed according to the procedure shown in Fig. (1), which uses collagen precipitation from sodium chloride solutions of various concentrations [28]. This procedure is often used to separate collagen types I-V. In our case, it was used to obtain fractions that were enriched in particular collagen types, so that it became possible to identify them. In order to minimize losses (for quantitation purposes), the isolation procedure was then simplified to include only one step (suspension in $4.5 \mathrm{~mol} / \mathrm{L} \mathrm{NaCl}+50 \mathrm{mmol} / \mathrm{L}$ Tris- $\mathrm{HCl}$, $\mathrm{pH}=7.5$; (Fig. (2)). $10 \mu \mathrm{L}$ of the internal standard stock solution $(1 \mathrm{mg} / \mathrm{mL})$ was added prior to analysis.

Cartilage samples: The cartilage was obtained from a porcine joint using a scalpel. The sample was lyophilized, frozen with liquid nitrogen and ground using a pestle and a mortar. The resulting powder was processed as shown in Fig. (2).

The fractions obtained by the processing described in Figs. (1) and (2) were cleaved with $\mathrm{CNBr}$ in $70 \%$ (v/v) formic acid under nitrogen. The samples were repeatedly dried (with a controlled warm air flow) and reconstituted in water (three times). After removing the $\mathrm{CNBr}$, the samples were reconstituted in $\mathrm{pH} 7.8,1 \mathrm{~mL} 0.05 \mathrm{~mol} / \mathrm{L}$ ammonium bicarbonate buffer and treated with trypsin (1:50 enzyme/substrate ratio) at $37^{\circ} \mathrm{C}$ for 3 hours.

\subsection{Total Protein Concentration}

The total protein concentration was determined by amino acid analysis using the Pico Tag method (acid hydrolysis of protein followed by RP-HPLC determination of amino acids after pre-column derivatization by phenylisothiocyanate) [29].

\subsection{Conditions for HPLC-MS/MS}

Chromatographic separation was carried out in a Jupiter Proteo 90 A column, 250 x 2 mm (Phenomenex, Torrance, CA, USA). The HPLC apparatus used was a HP 1100 LC system (Agilent, Palo Alto, CA, USA) consisting of a degasser, a binary pump, an autosampler, a thermostated column compartment and a diode array detector. It was coupled to an ion-trap mass spectrometer (Agilent LC-MSD Trap XCTUltra).

The separation was achieved via a linear gradient between mobile phase A (water-formic acid, $100: 0.03, \mathrm{v} / \mathrm{v}$ ) and $B$ (acetonitrile-formic acid, $100: 0.025 \mathrm{v} / \mathrm{v}$ ). Separation was started by running the system isocratically for two minutes with $2 \%$ mobile phase $\mathrm{B}$, followed by a gradient elution to $35 \% \mathrm{~B}$ at 40 minutes. Finally, the column was eluted with $100 \%$ B for 10 minutes. Equilibration before the next run 


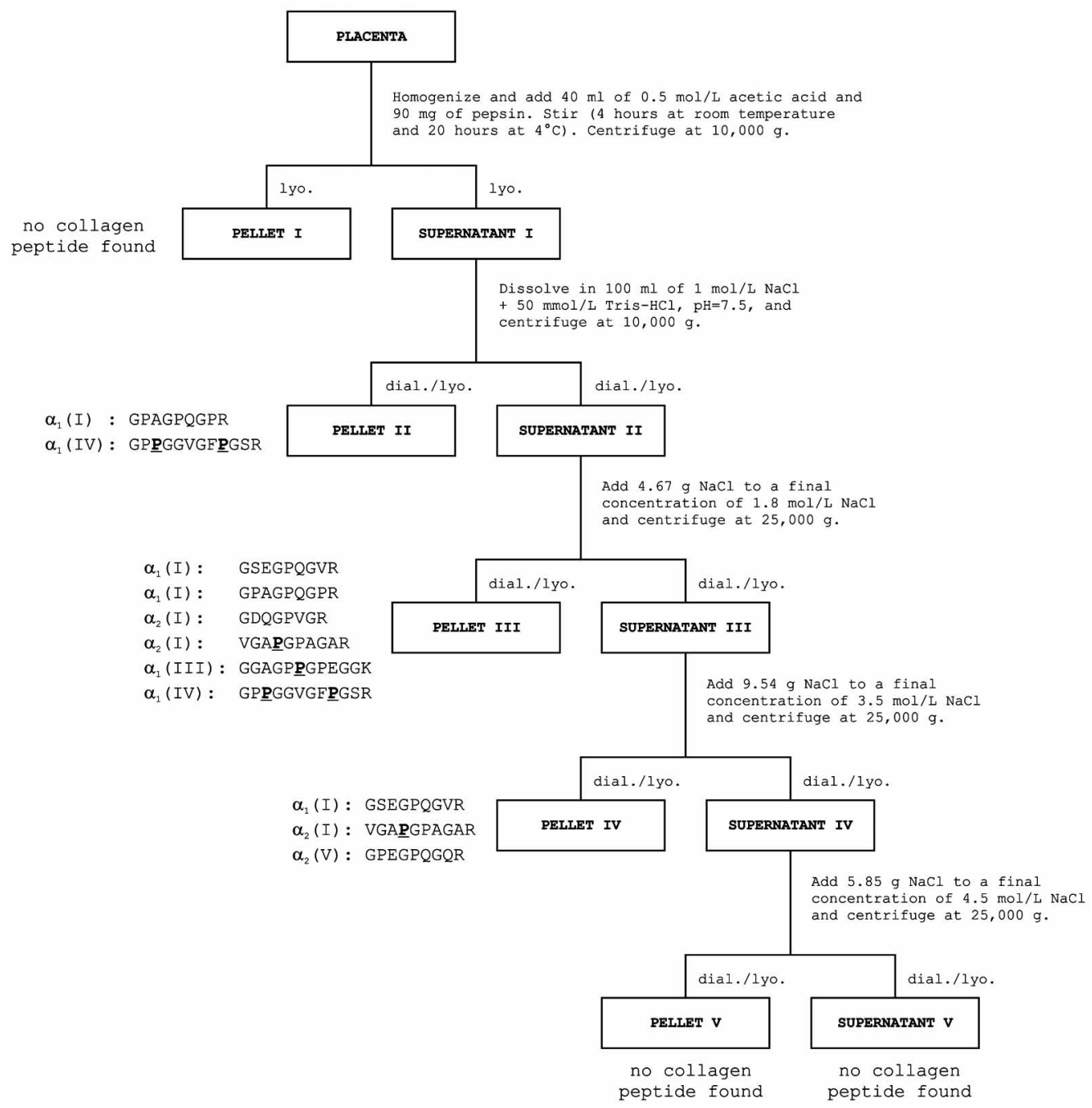

Fig. (1). Scheme for identification of collagen types in placenta.

was achieved by washing with $2 \%$ mobile phase B for 10 minutes. The flow-rate was $0.25 \mathrm{~mL} / \mathrm{min}$, injection volume was $40 \mu \mathrm{L}$, and the column temperature was held at $25^{\circ} \mathrm{C}$.

Atmospheric pressure ionization-electrospray ionization (API-ESI) positive mode ion-trap mass spectrometry was used. Operating conditions: drying gas $\left(\mathrm{N}_{2}\right), 10 \mathrm{~L} / \mathrm{min}$; drying gas temperature, $350^{\circ} \mathrm{C}$; nebulizer pressure, $172.37 \mathrm{kPa}$.

For peptide selection experiments, ions were observed over the mass range $\mathrm{m} / \mathrm{z} 100-2200$ (MS - standard mode, MS/MS - enhanced mode). Analysis was done in auto MS/MS mode (10 precursor ions, excluded after 2 spectra for $0.5 \mathrm{~min}$ ). The analyses of selected peptides were performed in multiple reaction monitoring mode (precursor ions were selected with respect to the peptides studied - see Results). The fragmentation amplitude was set to $1.14 \mathrm{~V}$.

Analysis of MS/MS data (peptide/protein identification and searching for possible post-translational modifications) was carried out using SpectrumMill software (v.3.02, Agilent). Searches were performed in the full protein databases
SwissProt and NCBInr and then on the data extracted from these databases.

\subsection{Determination of Detection and Quantitation Limits}

The limits of detection and quantitation for each marker peptide were determined from the calibration curve as $3 \mathrm{~N} / \mathrm{m}$ and $10 N / m$, respectively, where $N$ is the noise and $m$ is slope of the calibration curve (height of the peak vs. concentration). The values obtained were then recalculated for the whole chains using their molecular weights.

\section{RESULTS}

\subsection{Marker Peptide Selection}

The fractions obtained by processing placenta samples (see Fig. (1)) were analyzed for the presence of peptides unique to type I, III, IV, and V collagens. The cartilage sample was used for the identification of the characteristic peptides of type II collagen. The analyses showed that each collagen chain can be represented by at least one marker 


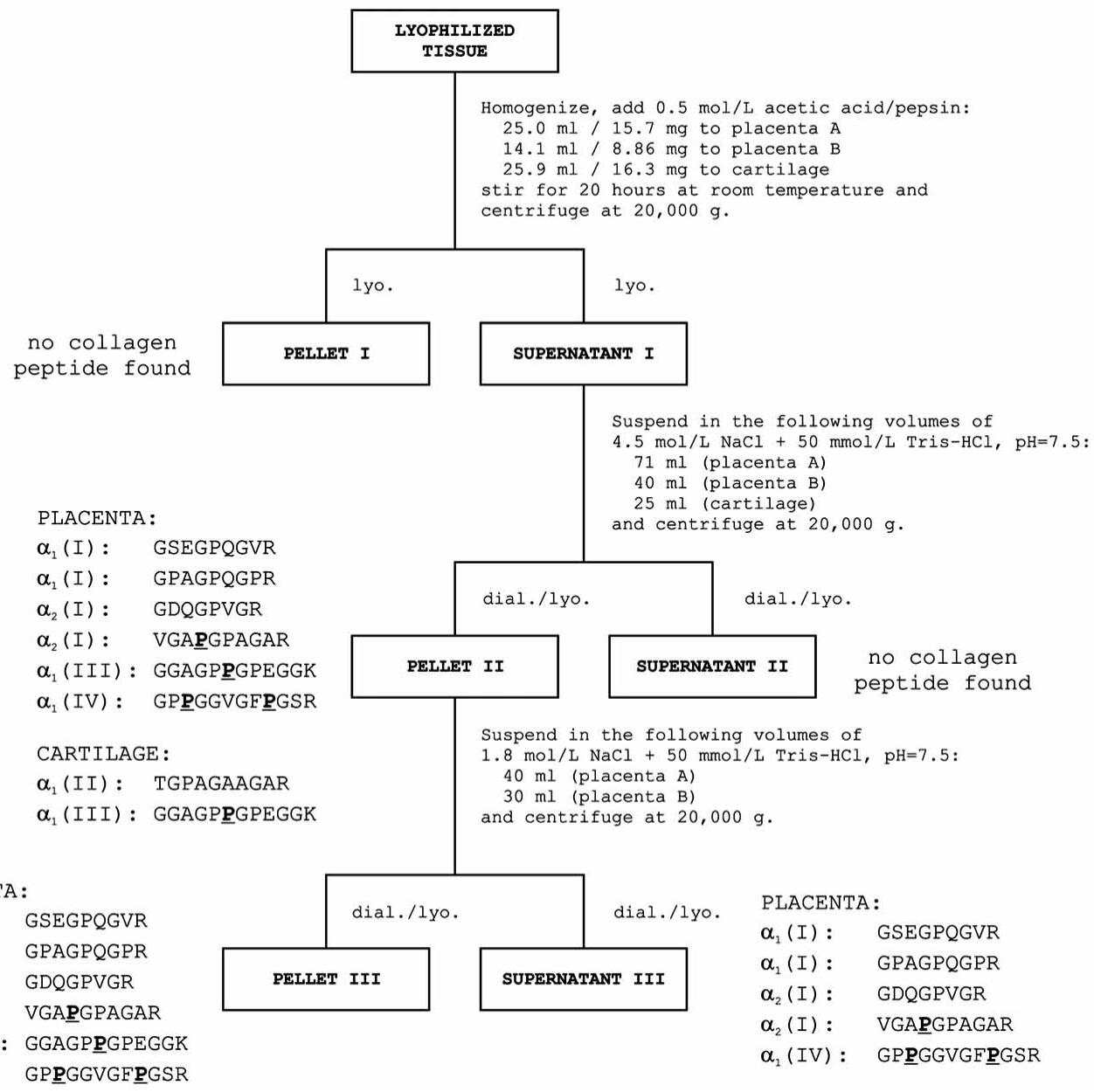

Fig. (2). Isolation of collagen types for quantification purposes.

peptide. The peptides selected as marker peptides had to meet all of the following criteria at the same time:

a) once detected in one sample, they had to be detected in all samples of a given type,

b) they had to be part of the collagenous domain,

c) they had to be $\mathrm{CNBr}$ /tryptic peptides, i.e. peptides obtained by cleavage with $\mathrm{CNBr} /$ trypsin at the specific sites,

d) their peak area in HPLC-MS/MS chromatograms of the standard samples had to be reproducible,

e) they had to be unique to a given collagen type, i.e. the peptide could not be part of any other protein structure in the SwissProt/NCBInr databases,

f) they had to be common to as many species as possible (preferably to human, bovine, and rat).

The presence of collagen marker peptides in particular fractions is illustrated in Figs. (1) and (2). The retention times and masses of both parent and product ions for $\alpha 1$ chains of type I - IV, and $\alpha 2$ chains of type I and V collagen marker peptides are summarized in Table $\mathbf{1}$.
The structures of all marker peptides were confirmed by HPLC-MS/MS (Figs. (3A) and (3B)). According to the Swiss-Prot database, each marker peptide was present only once per collagen chain for bovine, human and rat samples.

\subsection{HPLC-MS/MS Analyses of Samples}

Two types of tissue (placenta and cartilage) were analyzed for their content of collagen types I-V. Furthermore, the total protein concentration was determined for both the placenta and cartilage samples.

$\alpha 1(\mathrm{I}), \alpha 2(\mathrm{I}), \alpha 1(\mathrm{III})$, and $\alpha 1(\mathrm{IV})$ collagen chains were detected in the placenta when using the preparation procedure shown in Fig. (2). Types II and V (i.e. $\alpha 1$ (II) and $\alpha 2(\mathrm{~V})$ chains) were not detected. The $\alpha 2(\mathrm{~V})$ collagen chain was detected only when analyzing pellet IV prepared as shown in Fig. (1). However, this is not surprising, because pellet IV (Fig. (1)) contains only collagen type $\mathrm{V}$ and a fraction of the total amount of collagen type I. Therefore, it is to be expected that the concentration of collagen type $\mathrm{V}$ in pellet IV will be higher when compared to the more complex mixture in pellet II (Fig. (2)), where all collagen chains are expected. 
Table 1. List of Characteristic Marker Peptides for Collagen Types I - V

\begin{tabular}{|c|c|c|c|c|c|c|}
\hline Peptide & $\begin{array}{l}\text { Collagen } \\
\text { Chain }\end{array}$ & $\begin{array}{l}\text { HPLC Retention } \\
\text { Time [min] }\end{array}$ & $\begin{array}{l}\text { Charge } \\
\text { State }\end{array}$ & Precursor $\mathrm{MH}^{+}$ & Precursor Mass & Product Mass \\
\hline GSEGPQGVR & \multirow[t]{2}{*}{$\alpha_{1}(\mathrm{I})$} & 13.7 & $2+$ & 886.3 & 443.7 & 613.3 \\
\hline GPAGPQGPR & & 13.7 & $2+$ & 836.4 & 418.7 & 611.4 \\
\hline GDQGPVGR & $\alpha_{2}(\mathrm{I})$ & 13.0 & $2+$ & 785.3 & 393.2 & 485.3 \\
\hline TGPAGAAGAR & $\alpha_{1}(\mathrm{II})$ & 12.7 & $2+$ & 828.3 & 414.7 & 335.8 \\
\hline GGAGPPGPEGGK & $\alpha_{1}(\mathrm{III})$ & 13.0 & $2+$ & 980.5 & 498.7 & 377.8 \\
\hline 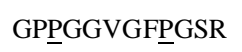 & $\alpha_{1}(\mathrm{IV})$ & 20.1 & $2+$ & 1116.5 & 558.8 & 962.6 \\
\hline GPEGPQGQR & $\alpha_{2}(\mathrm{~V})$ & 12.3 & $2+$ & 925.4 & 463.2 & 642.4 \\
\hline
\end{tabular}

A)
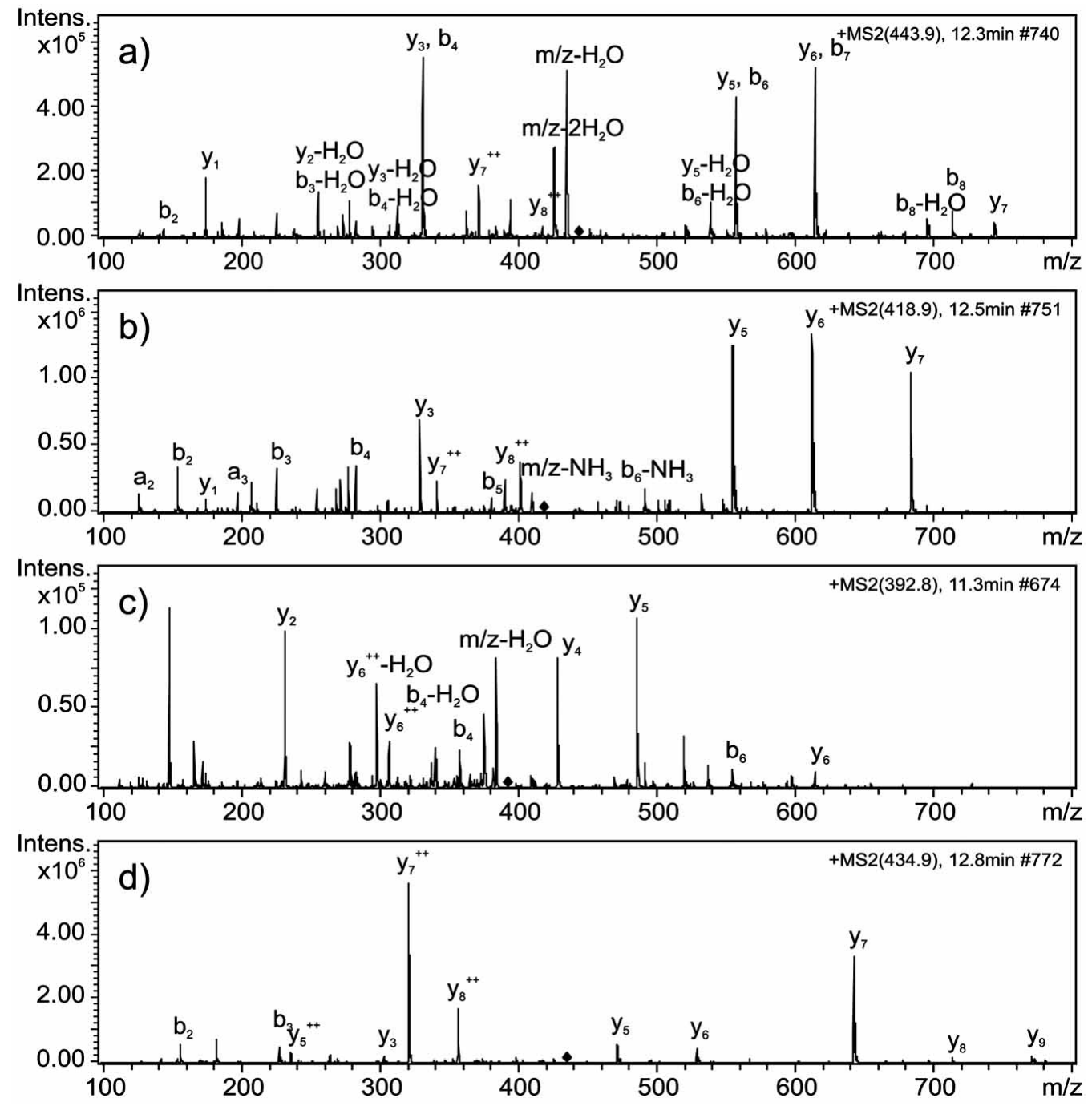
(Fig. 3) contd....

B)
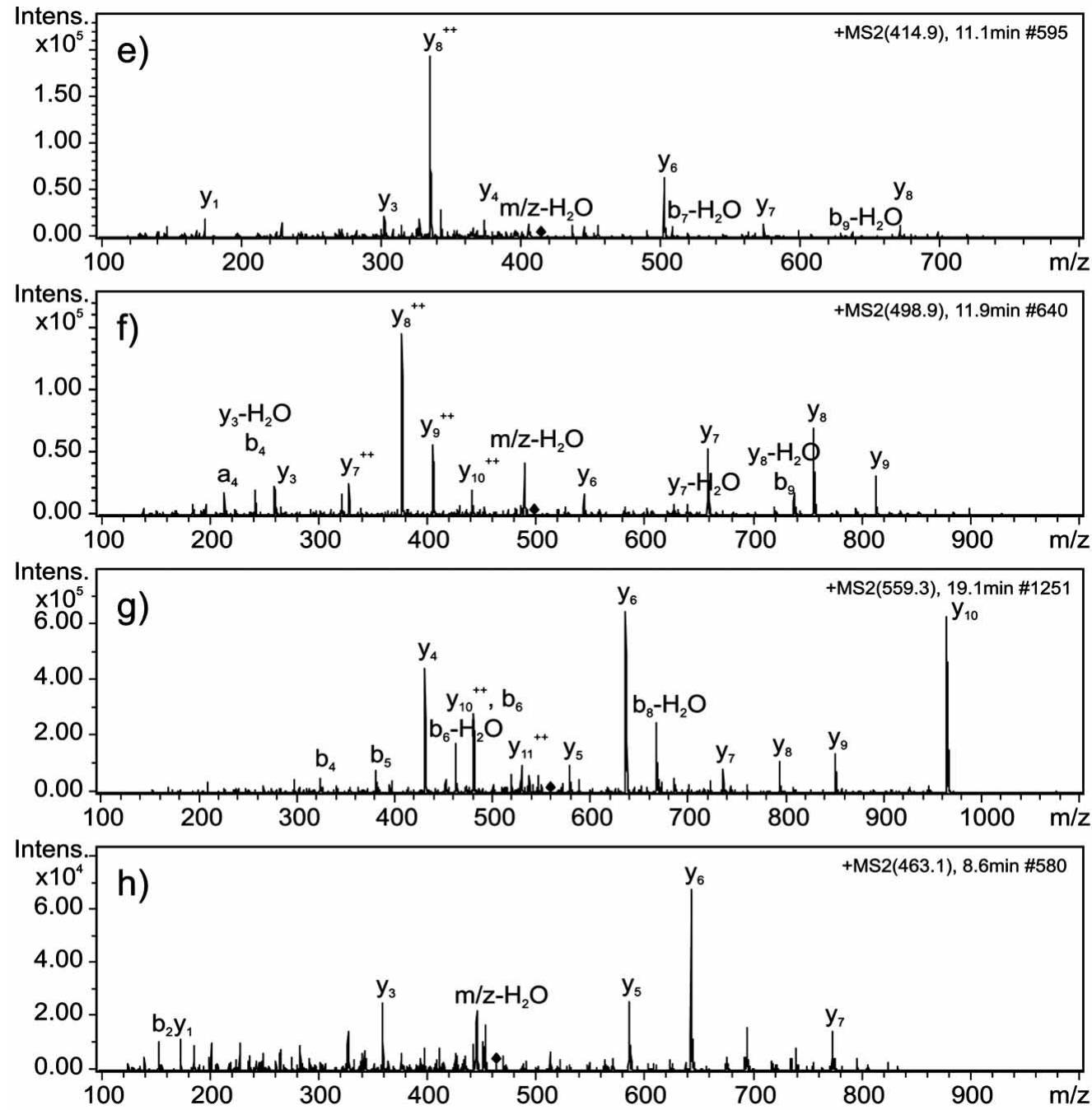

Fig. (3). A. MS/MS spectra of a) GSEGPQGVR, b) GPAGPQGPR, c) GDQGPVGR, and d) VGAPGPAGAR marker peptides found in placenta. B. MS/MS spectra of e) TGPAGAAGAR f) GGAGPPGPEGGK g) GPPGGVGFPGSR, and h) GPEGPQGQR marker peptides found in placenta and cartilage samples

The total protein concentration in pellet II (Fig. (2)) was found to be 94 and $96 \%$ (w/w) for placenta samples A and B, respectively. The cartilage samples had an average total protein concentration of $21 \%$.

In order to determine the ratios of collagen chains in tissues, the calibration mixtures of the GSEGPQGVR, GPAGPQGPR, GDQGPVGR, VGAPGPAGAR, TGPAGA

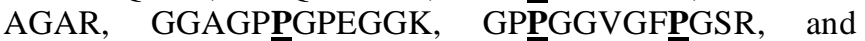
GPEGPQGQR peptides were analyzed and the results obtained were recalculated for the whole chains using the molecular weights of the collagen chains. The results are summarized in Table 2 . The molar concentrations and ratios obtained using the GSEGPQGVR and GPAGPQGPR peptides (both being part of the $\alpha 1$ (I) chain) were identical. Similarly, the same results were obtained for GDQGPVGR and VGAPGPAGAR, peptides that are part of the $\alpha 2(\mathrm{I})$ chain. The detection and quantitation limits (also recalculated to collagen chains) are summarized in Table $\mathbf{3}$.
The Swiss-Prot search revealed the presence of other proteins in both the placenta and cartilage samples, such as the fibronectin precursor for placenta and keratin type I and II for placenta and cartilage.

\section{DISCUSSION}

HPLC-MS/MS is a powerful tool not only for the identification, but also quantification of peptides and proteins. While the use of marker peptides has been previously employed by us for the identification and quantification of collagen types I and III in aorta, skin and tendon [1], the quantification of other collagen types (particularly II and IV) remained a problem, even when applying this method to a tissue where a higher content of these collagen types was expected. In fact, the direct cleavage of placenta or cartilage with $\mathrm{CNBr} /$ trypsin didn't provide satisfactory results. In this work, we have extended the use of HPLC-MS/MS to quantify other collagen types using marker peptides by modifying the sample preparation protocol. 
Table 2. Molar Ratios of Collagen Chains in Samples

\begin{tabular}{|c|c|c|c|c|c|}
\hline \multirow{2}{*}{ Sample } & \multicolumn{4}{|c|}{ Molar Ratio } \\
\cline { 2 - 6 } & $\boldsymbol{\alpha}_{\mathbf{1}}(\mathbf{I})$ & $\boldsymbol{\alpha}_{2}(\mathbf{I})$ & $\boldsymbol{\alpha}_{\mathbf{1}}(\mathbf{I I})$ & $\boldsymbol{\alpha}_{\mathbf{1}}(\mathbf{I I I})$ & $\boldsymbol{\alpha}_{\mathbf{1}}(\mathbf{I V})$ \\
\hline \hline Placenta A $(n=3)$ & $0.462 \pm 0.010$ & $0.229 \pm 0.007$ & N/D & $0.028 \pm 0.003$ & $0.281 \pm 0.019$ \\
\hline Placenta B $(n=3)$ & $0.338 \pm 0.034$ & $0.135 \pm 0.009$ & N/D & $0.024 \pm 0.009$ & $0.511 \pm 0.028$ \\
\hline Cartilage $(n=3)$ & N/D & N/D & $0.479 \pm 0.027$ & $0.521 \pm 0.027$ \\
\hline
\end{tabular}

$n=$ number of samples

Table 3. Detection and Quantitation Limits for Collagen Chains

\begin{tabular}{|c|c|c|c|c|c|c|}
\hline \multirow{2}{*}{ Characteristic } & \multicolumn{5}{|c|}{ Collagen Chain } \\
\cline { 2 - 7 } & $\boldsymbol{\alpha}_{\mathbf{1}}(\mathbf{I})$ & $\boldsymbol{\alpha}_{\mathbf{2}}(\mathbf{I})$ & $\boldsymbol{\alpha}_{\mathbf{1}}(\mathbf{I I})$ & $\boldsymbol{\alpha}_{\mathbf{1}}(\mathbf{I I I})$ & $\boldsymbol{\alpha}_{\mathbf{1}}(\mathbf{I V})$ & $\boldsymbol{\alpha}_{2}(\mathbf{V})$ \\
\hline \hline LOD $[\mathrm{mg} / \mathrm{mL}]$ & $2.15 \times 10^{-6}$ & $5.35 \times 10^{-7}$ & $7.58 \times 10^{-8}$ & $1.10 \times 10^{-6}$ & $5.65 \times 10^{-7}$ & $4.98 \times 10^{-7}$ \\
\hline LOQ $[\mathrm{mg} / \mathrm{mL}]$ & $7.16 \times 10^{-6}$ & $1.78 \times 10^{-6}$ & $2.53 \times 10^{-7}$ & $3.67 \times 10^{-6}$ & $1.88 \times 10^{-6}$ & $1.66 \times 10^{-6}$ \\
\hline
\end{tabular}

It was found that using the $\mathrm{CNBr} /$ trypsin digestion of collagen types I-V leads to peptide mixtures containing, among others, peptides characteristic for a given collagen type. The versatility of the proposed method lies in the fact that each of these peptides is common to at least three species - human, bovine, and rat.

The usability of the proposed method was demonstrated by determining the content of collagen types in real tissues. The molar ratio of three main collagen chains found in placenta $-\alpha 1(\mathrm{I}): \alpha 2(\mathrm{I}): \alpha 1(\mathrm{IV})$ was found to be $2: 1: 1.5$ and $2.5: 1: 3.8$ for the A and B placenta tissues, respectively. The content of $\alpha 1$ (III) was very low (approx. $0.84 \% \mathrm{w} / \mathrm{w}$ of the total protein concentration). The molar ratio $\alpha 1(\mathrm{I}): \alpha 2(\mathrm{I})$ $=2$ showes that the type I collagen found in placenta $\mathrm{A}$ is predominantely composed of heterotrimeric molecules $[\alpha 1(\mathrm{I})]_{2} \alpha 2(\mathrm{I})$. For placenta B, the ratio of $\alpha 1(\mathrm{I}): \alpha 2(\mathrm{I})=2.5$, indicates an increased homotrimer content. As expected, only the $\alpha 1$ (II) and $\alpha 1$ (III) collagens were found in cartilage. Similar results were obtained using CE-MS/MS (data not shown).

The method developed here represents a simple way of determining and quantifying the various collagen types in tissues using HPLC-MS/MS. Therefore, it retains the advantages of other highly specific methods of quantitation while avoiding the problems associated with them.

\section{ACKNOWLEDGEMENTS}

This work was supported by the Grant Agency of the Czech Republic, grants Nos. 203/09/0675, 203/08/1428, the Center for Cardiovascular Research 1M0510, and by Research Project AV0Z50110509.

\section{ABBREVIATIONS}

$$
\begin{aligned}
& \text { Dial }=\text { Dialysis } \\
& \text { Lyo }=\text { Lyophilization }
\end{aligned}
$$

\section{REFERENCES}

[1] Pataridis, S.; Eckhardt, A.; Mikulíková, K.; Sedláková, P.; Mikšík, I. Identification of collagen types in tissues using HPLC-MS/MS. $J$. Sep. Sci., 2008, 31, 3483-3488.

[2] Myllyharju, J.; Kivirikko, K.I. Collagens, modifying enzymes and their mutations in humans, flies and worms. Trends Genet., 2004, 20,33-43.

[3] Prockop, D.J.; Kivirikko, K.I. Collagens: molecular biology, diseases, and potentials for therapy. Аnnu. Rev. Biochem., 1995, 64, 403-434.

[4] Uitto, J. Collagen polymorphism: isolation and partial characterization of $\alpha 1(\mathrm{I})$-trimer molecules in normal human skin. Arch. Biochem. Biophys., 1979, 192, 371-379.

[5] Birk, D.E.; Fitch, J.M.; Babiarz, J.P.; Linsenmayer, T.F. Collagen type $\mathrm{I}$ and type $\mathrm{V}$ are present in the same fibril in the avian corneal stroma. J. Cell. Biol., 1988, 106, 999-1008.

[6] Sandberg, M.M.; Hirvonen, H.E.; Elima K.J.M.; Vuorio, E.I. Coexpression of collagens II and XI and alternative splicing of exon 2 of collagen II in several developing human tissues. Biochem. J., 1993, 294, 595-602.

[7] Miles, C.A.; Sims, T.J.; Camacho, N.P.; Bailey, A.J. The role of the $\alpha 2$ chain in the stabilization of the collagen type I heterotrimer: a study of the type I homotrimer in oim mouse tissues. J. Mol. Biol., 2002, 321, 797-805.

[8] Sasaki, T.; Arai, K.; Ono, M.; Yamaguchi, T.; Furuta, S.; Nagai, Y. Ehlers-Danlos syndrome. A variant characterized by the deficiency of pro $\alpha 2$ chain of type I procollagen. Arch. Dermatol., 1987, 123, 76-79.

[9] Kojima, T.; Shinkai, H.; Fujita, M.; Morita, E.; Okamoto, S. Case report and study of collagen metabolism in Ehlers-Danlos syndrome type II. J. Dermatol., 1988, 15, 155-160.

[10] Hata, R.; Kurata, S.; Shinkai, H. Existence of malfunctioning proa2(I) collagen genes in a patient with a proo2(I)-chain-defective variant of Ehlers-Danlos syndrome. Eur. J. Biochem., 1988, 174, 231-237.

[11] Nicholls, A.C.; Osse, G.; Schloon, H.G.; Lenard, H.G.; Deak, S.; Myers, J.C.; Prockop, D.J., Weigel, W.R.; Fryer, P.; Pope, F.M. The clinical features of homozygous $\alpha 2$ (I) collagen deficient osteogenesis imperfecta. J. Med. Genet., 1984, 21, 257-262.

[12] Pihlajaniemi, T.; Dickson, L.A.; Pope, F.M.; Korhonen, V.R.; Nicholls, A.; Prockop, D.J.; Myers, J.C. Osteogenesis imperfecta: cloning of a pro- $\alpha 2$ (I) collagen gene with a frameshift mutation. $J$. Biol. Chem., 1984, 259, 12941-12944.

[13] Vouyouka, A.G.; Pfeiffer, B.J.; Liem, T.K.; Taylor, T.A.; Mudaliar, J.; Phillips, C.L. The role of type I collagen in aortic wall strength with a homotrimeric. J. Vasc. Surg., 2001, 33, 1263-1270. 
[14] Pfeiffer, B.J.; Franklin, C.L.; Hsieh, F.H.; Bank, R.A.; Phillips, C.L. $\alpha 2$ (I) collagen deficient oim mice have altered biomechanical integrity, collagen content, and collagen crosslinking of their thoracic aorta. Matrix Biol., 2005, 24, 451-458.

[15] Nemirovskiy, O.V.; Dufield, D.R.; Sunyer, T.; Aggarwal, P.; Welsch, D.J.; Mathews, W.R. Discovery and development of a type II collagen neoepitope (TIINE) biomarker for matrix metalloproteinase activity: from in vitro to in vivo. Anal. Biochem., 2007, 361, 93-101.

[16] Bailey, A.J.; Sims, T.J.; Le Lous, M.; Bazin, S. Collagen polymorphism in experimental granulation tissue. Biochem. Biophys. Res. Commun., 1975, 66, 1160-1165.

[17] Shuttleworth, C.A.; Forrest, L.; Jackson, D.S. Comparison of the cyanogen bromide peptides of insoluble guinea-pig skin and scar collagen. Biochim. Biophys. Acta, 1975, 379, 207-216.

[18] Junge, K.; Klinge, U.; Rosch, R.; Mertens, P.R.; Kirch, J.; Klosterhalfen, B.; Lynen, P.; Schumpelick, V. Decreased collagen type I/III ratio in patients with recurring hernia after implantation of alloplastic prostheses. Langenbecks Arch. Surg., 2004, 389, 17-22.

[19] Stegemann, H.; Stalder K. Determination of hydroxyproline. Clin. Chim. Acta, 1967, 18, 267-273.

[20] Verch, R.L.; Wallach, S.; Peabody, R.A. Automated analysis of hydroxyproline with elimination of non-specific reacting substances. Clin. Chim. Acta, 1979, 96, 125-130.

[21] Pødenphant, J.; Larsen, N.E.; Christiansen, C. An easy and reliable method for determination of urinary hydroxyproline. Clin. Chim. Acta, 1984, 142, 145-148.
[22] Jimenez, W.; Pares, A.; Cabelleria, J.; Heredia, D.; Bruguera, M.; Torres, M.; Rojkind, M.; Rodes, J. Measurement of fibrosis in needle liver biopsies: evaluation of a colorimetric method. Hepatology, 1985, 5, 815-818.

[23] Lopéz-de León, A.; Rojkind, M. A simple micromethod for collagen and total protein determination in formalin-fixed paraffinembedded sections. J. Histochem. Cytochem., 1985, 33, 737-743.

[24] Taşkiran, D.; Taşkiran, E.; Yercan, H.; Kutay F.Z. Quantification of total collagen in rabbit tendon by the Sirius red method Turk. $J$. Med. Sci., 1999, 29, 7-9.

[25] van der Loos, C.M.; Marijianowski, M.M.H.; Becker, A.E. Quantification in immunohistochemistry: the measurement of the ratios of collagen types I and II. Histochem. J., 1994, 26, 347-354.

[26] Deyl, Z.; Mikšík, I. Advanced separation methods for collagen parent $\alpha$-chains, their polymers and fragments. J. Chromatogr. B, 2000, 739, 3-31.

[27] Zhang, G.; Sun, A.; Li, W.; Liu, T.; Su, Z. Mass spectrometric analysis of enzymatic digestion of denatured collagen for identification of collagen type. J. Chromatogr. A, 2006, 1114, 274-277.

[28] Miller, E.J.; Rhodes, R.K. Preparation and characterization of the different types of collagen. Methods Enzymol., 1982, 82, 33-64.

[29] Mikulíková, K.; Mikšík, I.; Deyl, Z. Non-enzymatic posttranslational modifications of bovine serum albumin by oxo-compounds investigated by chromatographic and electrophoretic methods. $J$. Chromatogr. B, 2005, 815, 315-331. 